\title{
RESPOSTA DO ÁLAMO (Populus deltoides Marsh) À ADUBAÇÃO NITROGENADA EM DOIS SÍTIOS DO MUNICÍPIO DE SÃO MATEUS DO SUL, PARANÁ
}

\section{POPLAR (Populus deltoides Marsh) RESPONSE TO NITROGEN ADDITION AT TWO SITES IN SÃO MATEUS DO SUL, PR}

\author{
Giancarlo Mira Otto ${ }^{1}$ Antônio Carlos Vargas Motta ${ }^{2}$ Carlos Bruno Reissman ${ }^{3}$
}

\section{RESUMO}

Os plantios de álamo realizados na região de São Mateus do Sul, PR, não tem o desenvolvimento esperado, pela ausência de informações a respeito de clones ideais, pragas, doenças e nutrição. Diante da carência de estudos sobre fertilização de povoamentos de álamo para as condições em que a espécie é plantada no Brasil e visando a melhorar a produtividade dos plantios de álamo na região de São Mateus do Sul, PR, foi instalado um experimento testando cinco doses de $\mathrm{N}$ e mais um formulado NPK em dois sítios diferentes. As avaliações mostraram um pequeno efeito da adubação sobre o crescimento em circunferência à altura do peito (CAP) e altura total (Ht). Também houve correlação positiva entre os parâmetros de crescimento com P, Ca e pH do solo, e os teores de P, Ca e B da folha, indicando a influência desses elementos sobre o crescimento das plantas, sugerindo a necessidade de adequação da fertilidade do solo, via corretivos da acidez e adubação, antes da implantação das mudas.

Palavras chave: Nutrição do álamo; produção de madeira; florestamento.

\section{ABSTRACT}

Poplar plantations have shown a growth pattern well below their potential in Iguaçu River Basin, PR, Brazil. In addition, there is a lack of information about poplar nutrition and response to fertilizer application in the area, limiting fertilizer recommendation. This study evaluated the poplar response to $\mathrm{N}$ rates $(0,20,40$ e $\left.80 \mathrm{~kg} \mathrm{ha}^{-1} . \mathrm{yr}\right)$ and a combined effect with the application of $\mathrm{N}, \mathrm{P}_{2} \mathrm{O}_{5}$, and $\mathrm{K}_{2} \mathrm{O}\left(27,15\right.$, and $\left.11 \mathrm{~kg} \mathrm{ha}^{-1} . \mathrm{yr}\right)$, during three years after planting in two sites (lowland and dryland area). Better tree growth was observed at dryland area and fertilizer application provided a small improvement on circumference at breast high and total high in both sites. There was a positive correlation between soil properties $(\mathrm{P}, \mathrm{Ca}$, and $\mathrm{pH})$ and nutrient concentration in the leaves ( $\mathrm{P}, \mathrm{Ca}$ and $\mathrm{B}$ ) with plant growth. Results suggested the need of soil fertility adjustment, by acidity correctors and fertilizers, before tree planting.

Keywords: Poplar nutrition; wod production; implanted forests.

\section{INTRODUÇÃO}

A cultura do Álamo (Populus deltoides Marsh.) vem se difundindo no sul do estado do Paraná e no norte de Santa Catarina, tendo alcançado atualmente uma área aproximada de 5.000 ha, sempre nas várzeas da bacia do Médio Iguaçu. Esses plantios comerciais de Álamo tem como finalidade a fabricação de palitos de fósforos e laminados em geral e são muito jovens, pois ainda não se tem a experiência de uma rotação.

Diferentemente das coníferas, folhosas decíduas como o álamo necessitam de grandes quantidades de nutrientes e, estando ambas em sítios de fertilidade similar, são mais predispostas a deficiências (Brown, 1999). Em geral, álamos preferem solos de textura leve, permeáveis, profundos e úmidos, pH próximo da neutralidade, alta fertilidade, além de bom suprimento de água (Birler, 1994).

A fertilização tem um grande potencial de aumentar significativamente a produção em plantios de álamo (Van Oosten e Zabek, 2004), sendo N e P os maiores limitantes ao crescimento de Álamos híbridos (Brown, 1999; Van den Driessche, 2000). A importância do N no crescimento do álamo foi demonstrada por Brown e van den Driesche (2002) que, ao trabalharem com dois clones híbridos de álamo no Canadá, após

1. Engenheiro Florestal, Mestrando pelo Curso de Pós Graduação em Agronomia, Setor de Ciências Agrárias, Universidade Federal do Paraná, Rua Manoel Furtado Neves, 1118, CEP 83900-000, São Mateus do Sul (PR). ottogm@uol.com.br

2. Engenheiro Agrônomo, Dr., Professor do Departamento de Solos, Setor de Ciências Agrárias, Universidade Federal do Paraná, Rua dos funcionários, 1540, CEP 80035-050, Curitiba (PR). mottaacv@ufpr.br

3. Engenheiro Florestal, Dr., Professor do Departamento de Solos, Setor de Ciências Agrárias, Universidade Federal do Paraná, Rua dos funcionários, 1540, CEP 80035-050, Curitiba (PR).reissman@ufpr.br

Recebido para publicação em 28/02/2006 e aceito em 4/04/2007. 
duas estações de crescimento, obtiveram aumentos lineares no crescimento do tronco com a aplicação de $\mathrm{N}$ no plantio.

A resposta à adubação nitrogenada pode variar em função dos clones, visto que Johnson e Zhao (2004) encontraram respostas positivas para biomassa total, eficiência no uso do Nitrogênio e eficiência de área foliar em relação a diferentes doses de $\mathrm{N}$, sendo que os melhores desempenhos foram daqueles híbridos com mãe de Populus deltoides. Van Oosten e Zabek (2004) realizaram, no Canadá, um teste aplicando doses de NPK em plantios de 2 anos e após o décimo ano de idade os efeitos ainda eram evidentes, com diferenças significativas para a aplicação conjunta de $\mathrm{N}$ e P, sem resposta para o K.

Contudo, o uso de $\mathrm{N}$, sobretudo em grande quantidade, pode causar risco à produtividade, visto que Belanger et al. (1990) constataram que a fertilização com N isolado pode predispor as árvores a infecções pelo aumento da relação folha/raiz e aumentado o stress hídrico da planta. Da mesma forma, Harvey e Van den Driessche (1997), testando doses de N e P, descobriram que altas doses de N aumentam a suscetibilidade a embolia nos vasos do xilema em períodos de seca, que reduziram o crescimento, podendo ser amenizado quando da adição de P. Entretanto, o uso de adubações nitrogenadas e fosfatadas podem reduzir a atividade micorrízica como constatada em Populus trichocarpa e em Populus tremula x tremuloides (Baum e Makeschin, 2000).

Os teores foliares de $\mathrm{N}$ podem dar indicativo sobre resposta da cultura, sendo que valores entre $20 \mathrm{e}$ $30 \mathrm{~g} \mathrm{~N} \mathrm{~kg}^{-1}$ na folha representam níveis adequados para o álamo, dentro dos quais a resposta á adubação é incerta (Stanturf et al., 2001). Na avaliação do estado nutricional das plantas, a análise foliar tem se mostrado um excelente ferramenta, especialmente se são comparadas árvores vigorosas e de baixo crescimento (Gonçalvez 1997). Contudo, Brown (1999) cita que aplicar níveis críticos é problemático, pois esses níveis críticos associados com baixo crescimento podem variar com a idade e com a espécie e diminuir com a idade da planta, além de variar com a disponibilidade de outros recursos, como umidade e outros nutrientes. Em função disso, indica a utilização de relações entre nutrientes, especialmente N/K, N/P e até N/S como elemento de diagnóstico.

Relações entre N, P, K, Ca e Mg também são citados por Gonçalvez (1997) como uma ferramenta mais útil para diagnóstico de estresse nutricional de povoamentos florestais do que níveis críticos individuais foliares. Stanturf et al. (2001), todavia, cita que consumo de luxo de N pode afetar a acuracidade das taxas e interpretação dessas relações, dificultando a determinação de qual nutriente é deficiente.

Pela ausência de informações nutricionais do Álamo para as condições brasileiras, o presente trabalho tem como objetivo principal fornecer subsídios sobre adubação (N e NPK + Ca, Mg e S) e aspectos nutricionais do álamo em dois sítios.

\section{MATERIAIS E MÉTODOS}

\section{Local}

O experimento foi instalado em áreas de plantio de álamo localizadas no município de São Mateus do Sul, PR, na bacia do Rio Iguaçu, com coordenadas $25^{\circ} 54^{\prime}$ S e $50^{\circ} 24^{\prime}$ W. O clima da região é Subtropical Úmido Mesotérmico, tipo CfB (Köeppen), com várias e severas geadas por ano. A precipitação é de 1600$1700 \mathrm{~mm} / \mathrm{ano}$, regularmente distribuída e altitude média é de $750 \mathrm{~m}$.

O experimento foi instalado em duas áreas distintas quanto ao manejo anterior e tipo de solo, distantes aproximadamente $500 \mathrm{~m}$ entre si, sendo elas:

\section{Área de encosta cultivada com culturas agrícolas}

O solo dessa área de estudo foi classificado como Cambissolo Vermelho Amarelo álico epieutrófico de textura argilosa (Rocha et al., 1999) (CA). O solo vinha sendo cultivado por aproximadamente 10 anos, para produção de soja e milho, em sistema de plantio convencional, com uso limitado de adubo e calcário. A cultura anterior de verão, 2001/2002, foi soja em plantio direto.

\section{Área de várzea úmida do rio Iguaçu sem prévio cultivo.}

A área era composta por Solo Orgânico Sáprico álico epieutrófico (SO), com vegetação natural de campo. O solo era sujeito a alagamentos freqüentes e com lençol freático em torno de 0,5 $\mathrm{m}$ de profundidade. 
Para implantação do álamo, a área foi drenada, com abertura de canais em intervalos de $40 \mathrm{~m}$ e $1,5 \mathrm{~m}$ de profundidade. Após drenagem, a área recebeu apenas calagem de 5 t/ha com o calcário calcítico e incorporação a $20 \mathrm{~cm}$ de profundidade com grade aradora. Após, nas linhas de plantio, foi levantado um camalhão de aproximadamente $0,4 \mathrm{~m}$ de altura e $1 \mathrm{~m}$ de largura, feito com um terraceador, que retirou horizonte A das proximidades para diminuir o efeito das enchentes.

\section{Instalação do experimento}

Após o preparo do solo, em final de julho de 2002, foi realizado o plantio das mudas, em forma de estacas de $4,5 \mathrm{~m}$ de altura e $5 \mathrm{~cm}$ de diâmetro a altura do peito (DAP). As covas foram feitas mecanizadamente com um perfurador de solo com dimensões de $1 \mathrm{~m}$ de profundidade e $20 \mathrm{~cm}$ de diâmetro. Em mistura com o solo, no terço médio da cova, foram colocados $3 \mathrm{~kg}$ de esterco sólido de gado confinado curtido e, no terço superior mais $1 \mathrm{~kg}$ de cinza de caldeira. Na área de encosta, a implantação ocorreu sobre $\mathrm{o}$ triticale plantado, que estava aproximadamente com $25 \mathrm{~cm}$ de altura e o espaçamento foi de $6 \times 6 \mathrm{~m}$, totalizando 278 árvores/ha. Na várzea, o espaçamento foi de 6 x 5,2 m, totalizando 320 árvores ha¹

As parcelas experimentais eram compostas por quatro linhas, cada uma com seis plantas, totalizando 24 plantas por parcela, sendo marcadas oito plantas úteis, quatro de cada linha interna, para obtenção de dados de crescimento e material de planta para verificação de aspectos nutricionais.

Adubação nitrogenada foi aplicada em coroa de $0,5 \mathrm{~m}$ de raio ao redor da planta, parcelada em duas vezes, uma em 15/12/2002 e a segunda em 4/02/2003. A mesma adubação foi repetida no segundo e terceiro períodos de crescimento, com parcelamentos em 10/11/03 e 12/11/04 e também em 15/112005 e 12/12/2005. Os tratamentos utilizados foram as doses de $\mathrm{N}$ foram 0 (testemunha), 20, 40 e $80 \mathrm{~kg}$ ha.ano-1, aplicados na forma de uréia, mais a adubação completa, que foi uma combinação de $\mathrm{N}, \mathrm{P}_{2} \mathrm{O}_{5}$ e $\mathrm{K}_{2} \mathrm{O}(27,15$ e $11 \mathrm{~kg} \mathrm{ha}^{-1}$ respectivamente, mais $\mathrm{Ca}, \mathrm{Mg}$, e S).

\section{Coleta de dados}

\section{Circunferência a altura do peito (CAP) e altura total (Ht)}

Para eliminar diferenças de tamanho das mudas provenientes do viveiro, foram medidas a CAP e a altura total (Ht) da muda antes da aplicação dos tratamentos, e, após a estação de crescimento, foi feita uma nova medição. O experimento foi acompanhado durante três períodos de crescimento (2002/2003, 2003/2004 e 2004/2005). Os valores utilizados na compilação dos dados foram a diferença entre o crescimento inicial e o final. As medidas das circunferências foram tomadas com fita métrica e as alturas com uma régua de $5 \mathrm{~m}$. Como o experimento foi instalado em dezembro de 2002, a primeira medição de CAP e Ht foi realizada junto com a primeira adubação. A medição após o primeiro período vegetativo foi em 8/08/2003. A terceira medição ocorreu em 24/04/2004 e a quarta em 25/08/2005. Os valores utilizados foram as médias totais de todas as árvores medidas.

\section{Análise de solos e foliares}

As amostras de solo foram coletadas de cada bloco, na forma de amostra composta, na profundidade de 0-20 cm. Foram feitas analises de $\mathrm{MO}, \mathrm{pH} \mathrm{CaCl}_{2}$ 0,01 M, P-resina, Ca, Mg, K, H+Al, Al, e os micronutrientes B, Cu, Fe, Mn e Zn segundo os métodos descritos por Raij e Quaggio (1983). Na Tabela 1, são apresentados os resultados da análise dos solos do local do experimento.

No final de janeiro de 2003 e 2004, quando as folhas estavam maduras, foram coletadas dez unidadesde cada árvore, de posição intermediária de galhos do terço médio da copa, posicionados na face norte da planta. A análise foliar para N, P, K, Ca, Mg, Cu, Zn, Fe, Mn e B foi realizada nos dois primeiros anos seguindo metodologia descrita por Malavolta (1989). 
TABELA 1: Analise de solo do experimento de adubação de álamo no município de São Mateus do Sul, PR. TABLE 1: Soil analysis of poplar fertilization in São Mateus do Sul, PR.

\begin{tabular}{|c|c|c|c|c|c|c|c|c|c|c|c|}
\hline & $\begin{array}{l}\text { P resina } \\
\text { mg.dm } \\
-3\end{array}$ & $\begin{array}{c}\mathrm{MO} \\
\text { g. } \mathrm{dm}^{-3}\end{array}$ & $\begin{array}{c}\mathrm{pH} \\
\mathrm{CaCl}_{2}\end{array}$ & $\mathrm{H}+\mathrm{Al}$ & $\mathrm{Al}$ & $\begin{array}{l}\mathrm{K} \\
\mathrm{cm}\end{array}$ & $\begin{array}{l}\mathrm{Ca} \\
\mathrm{mm}^{-3}\end{array}$ & $\mathrm{Mg}$ & CTC & $\mathrm{V}$ & $\begin{array}{l}\mathrm{Al} \\
\%\end{array}$ \\
\hline CA & 9 & 49 & 4,6 & 9,8 & 2,2 & 0,39 & 5,3 & 1,1 & 16,6 & 44 & 18,8 \\
\hline SO & 8 & 102 & 4 & 18,5 & 4,4 & 0,15 & 2,1 & 0,6 & 21,4 & 13 & 60,5 \\
\hline & \multicolumn{2}{|c|}{ Boro } & \multicolumn{2}{|c|}{ Cobre } & \multicolumn{3}{|c|}{ Ferro } & \multicolumn{2}{|c|}{ Manganês } & \multicolumn{2}{|c|}{ Zinco } \\
\hline & \multicolumn{11}{|c|}{$\mathrm{mg} \mathrm{dm^{-3 }}$} \\
\hline CA & \multicolumn{2}{|c|}{0,50} & \multirow{2}{*}{\multicolumn{2}{|c|}{2}} & \multicolumn{3}{|c|}{92} & \multicolumn{2}{|c|}{91} & \multicolumn{2}{|c|}{1,9} \\
\hline SO & \multicolumn{2}{|c|}{0,35} & & 1 & \multicolumn{3}{|c|}{239} & \multicolumn{2}{|c|}{4,8} & \multicolumn{2}{|c|}{1,0} \\
\hline
\end{tabular}

Em que: $\mathrm{MO}$ = matéria orgânica; $\mathrm{CTC}$ = capacidade de troca de cátions; $\mathrm{V} \%$ = saturação de bases; $\mathrm{Al} \%$ = saturação de alumínio; $\mathrm{CA}$ = cambissolo; $\mathrm{SO}$ = solo orgânico.

\section{Análise estatística}

O experimento foi analisado segundo um delineamento com tratamento fatorial de blocos ao acaso, sendo o primeiro fator a adubação, com cinco níveis, que consistia de doses de $\mathrm{N}$ e tratamento adicional de adubação completa com NPK, com quatro repetições O segundo fator foi o solo, com dois níveis. Cada tratamento consistia de um nível de adubação com $\mathrm{N}$ combinado com um tipo de solo.

Os resultados foram submetidos à análise de variância. Inicialmente foram avaliados quanto à homogeneidade de variâncias pelo teste de Bartlett, sendo utilizada a transformação de dados quando necessário. As variáveis que revelaram diferenças significativas foram testadas pelo teste de " $\mathrm{t}$ " ao nível de 5\% de probabilidade. Foi realizada também a análise de regressão entre as variáveis dose de N e CAP.

\section{RESULTADOS E DISCUSSÃO}

\section{Circunferência à altura do peito (CAP) e altura total (Ht)}

Os resultados obtidos indicam um aumento na taxa de crescimento com idade do plantio nos três anos avaliados, independente dos tratamentos e sítios utilizados. No sítio CA, as médias de incremento foram de 3,8 a 4,9 cm em CAP no primeiro ano e 16,0 a 21,1 cm em CAP após 3 anos. Já no sítio SO os incrementos em CAP foram de 1,8 a 2,9 cm e 14,2 a 16,7 cm, para o primeiro ano e após 3 anos respectivamente. Contudo, os resultados obtidos indicaram que a adubação nitrogenada proporcionou pequeno acréscimo em CAP nos dois sítios (Figura 1).

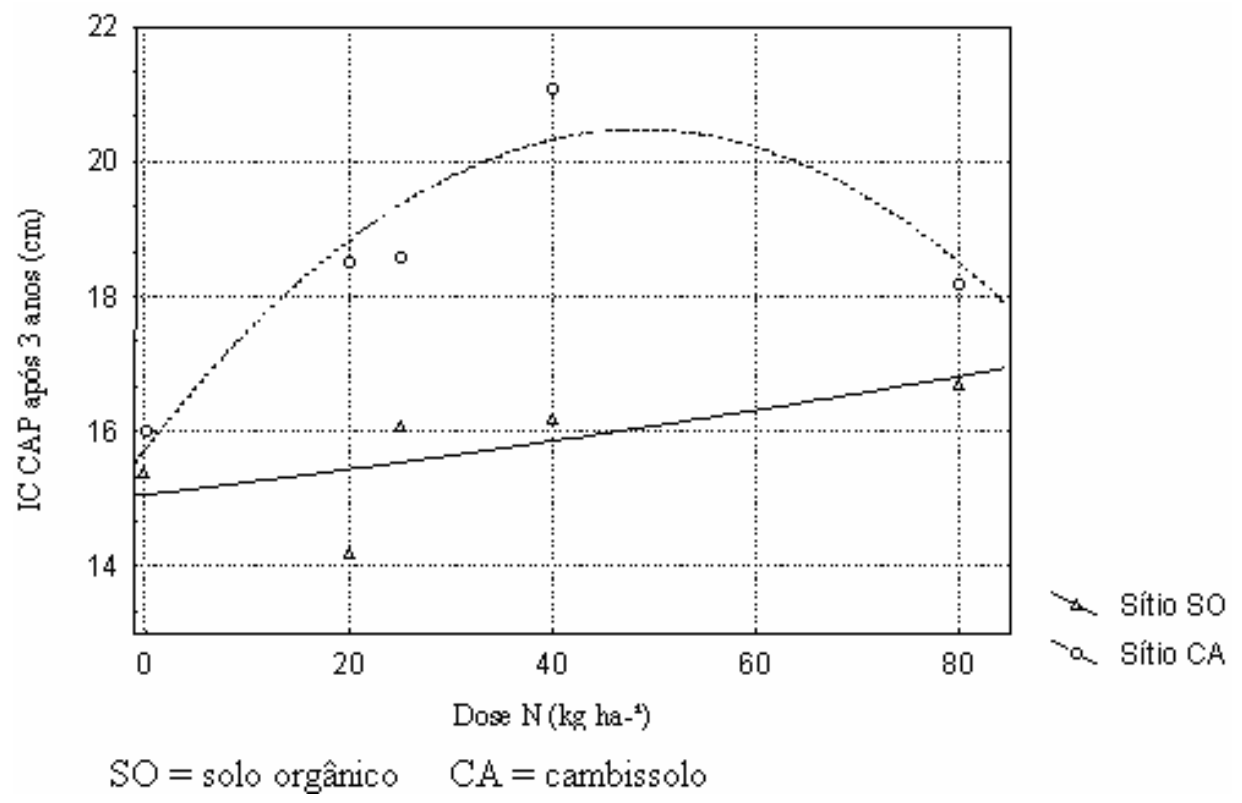

FIGURA 1: Incremento corrente em CAP de álamo após 3 anos em relação à adição de N.

FIGURE 1: DBH current increment after 3 years in relation to $\mathrm{N}$ addition. 
O mesmo fato ocorreu na altura de planta, em que os incrementos variaram de 0,35 a 0,51 e 3,07 a 3,50 m para o primeiro e após 3 anos respectivamente no CA. Já em SO as alturas variaram de 0,25 a 0,3 m e 2,47 a 2,71 m para o primeiro ano e após o terceiro ano respectivamente. Corroborando com resultados aqui obtidos, diversos autores indicam o $\mathrm{N}$ é como sendo o principal nutriente para o estabelecimento dos plantios de álamo, havendo respostas à sua adição (Jobling, 1990; Ente Nazionale per la Cellulosa e per la Carta, 1987; Stanturf et al, 2001; Dickmann et al, 2001; Van Den Driesshe, 1999; Brown e Van Den Driesshe, 2002, Johnson e Zhao, 2004).

Contudo, os acréscimos observados podem ser considerados pequenos, e deve-se provavelmente ao elevado teor de matéria orgânica do solo utilizado (Tabela 1), sobretudo no que se refere ao solo orgânico de várzea (SO), que possibilita uma elevada liberação de N. Ainda, a passagem de condição anaeróbia para aeróbia com drenagem do solo orgânico pode ter propiciado uma elevada decomposição nos primeiros anos, o que ocasionaria uma grande liberação de N (Canellas et al., 1999). Não pode ainda ser descartada a possibilidade de haver outros fatores limitantes ao crescimento da planta, afetando negativamente a resposta à adubação nitrogenada.

Apesar da pequena resposta aos tratamentos, verificou-se, contudo, uma grande variação no desenvolvimento das plantas, entre sítios utilizados (Figura 1).

Logo, os resultados obtidos se contrapõem à idéia de que os melhores sítios de crescimento do álamo são solos de várzea, visto que os maiores crescimentos foram obtidos no solo de encosta (CA) mais elevado (Figura 1). Isso pode ser explicado por esse solo ter sido cultivado anteriormente com soja e milho, o que lhe confere melhores características físicas e químicas. Também tem naturalmente uma melhor profundidade efetiva para as raízes explorarem, o que não ocorre no SO que, além de uma fertilidade menor, pode ter problemas para um enraizamento profundo, em virtude de acidez excessiva, lençol freático alto e camadas de impedimento (gleização).

Assim, os resultados sugerem que a escolha de solos de várzea para implantação de álamo em regiões semiáridas como Chile e parte dos países mediterrâneos, não se aplica às condições do centro sul do Paraná onde elevada precipitação pode fornecer água suficiente para crescimento das plantas.

No geral, o desenvolvimento foi baixo comparado aos sítios estabelecidos por Birler (1994). O autor estabeleceu índices de sítio para um clone euroamericano na Turquia, e para o sitio 3 (de 1 a 4, em ordem decrescente de qualidade) o incremento nesse mesmo espaçamento e período de crescimento deveria ser de $30 \mathrm{~cm}$ em CAP e $5 \mathrm{~m}$ em $\mathrm{H}$. Sendo assim, os resultados aqui obtidos podem ser comparáveis a um sítio de nível 4.

\section{Teores foliares de macronutrientes (N, P, K, Ca, Mg e S)}

Os resultados mostraram que houve diferença na concentração foliar de $\mathrm{N}$ para os diferentes tratamentos apenas no primeiro ano de aplicação, enquanto que no segundo ano, as concentrações não diferiram significativamente talvez por um efeito de diluição, motivado pela maior massa foliar. É importante considerar que até $50 \%$ do $\mathrm{N}$ utilizado pelas plantas provêm de ciclagem interna ocorrida no outono anterior (Ericsson et al., 1992) e sendo as mudas utilizadas provenientes de uma condição de excelente nutrição no viveiro, é provável que uma grande reserva de $\mathrm{N}$ nas mudas tenha contribuído para a não-diferença no primeiro ano.

Os teores de $\mathrm{N}$ variaram de 23,4 a 27,9 $\mathrm{g} \mathrm{kg}^{-1}$ nos dois anos avaliados , estando tais valores de $\mathrm{N}$ entre 20 e $30 \mathrm{~g} \mathrm{~kg}^{-1}$, indicados como níveis adequados para a planta, dentro dos quais a resposta à adubação é incerta (Stanturf et al., 2001). Sendo assim, os valores de $\mathrm{N}$ encontrados indicam que o seu suprimento pelos solos avaliados foi suficiente para satisfazer a cultura, e o baixo desenvolvimento deu-se possivelmente por outros fatores, como Ca, pH, e P. Com base nas relações N/K, N/P e até N/S proposta por Brown (1999), o nível de $\mathrm{N}$ encontra-se relativamente alto, sugerindo uma deficiência de outros elementos, especialmente $\mathrm{P}$ (Tabela 2). 
TABELA 2: Relações N/P foliares de álamo para primeiro e segundo anos, em relação às diferentes doses de adubação.

TABLE 2: N/P foliar poplar relations for first and second years, in relation to different fertilization levels.

\begin{tabular}{l|c|c|ccc|c}
\hline Época & Sitio & $80 \mathrm{~N}$ & $40 \mathrm{~N}$ & $25: 17: 11 \mathrm{NPK}$ & $20 \mathrm{~N}$ & $00 \mathrm{~N}$ \\
\hline \multirow{2}{*}{$1^{\circ}$ ano } & CA & 19,3 & 18,9 & 18,9 & 18,0 & 17,6 \\
& SO & 19,9 & 20,0 & 18,8 & 19,7 & 18,6 \\
\hline \multirow{2}{*}{$2^{\circ}$ ano } & CA & 19,4 & 18,1 & 21,7 & 16,7 & 18,5 \\
& SO & 19,9 & 18,8 & 16,7 & 17,9 & 19,0 \\
\hline
\end{tabular}

Em que: $\mathrm{CA}$ = cambissolo; $\mathrm{SO}$ = solo orgânico.

A limitação do P sobre o crescimento da planta ficou confirmada com boa relação via regressão entre $\mathrm{P}$ disponível do solo e teor de $\mathrm{P}$ foliar com o incremento em CAP combinando ambos sítios [Incremento CAP $1^{\circ}$ ano $(\mathrm{cm})=-12,0+11,8 \mathrm{P}_{\text {foliar, }} \mathrm{R}^{2}=51 \%$ ] e altura [Incremento $\mathrm{Ht} 1^{\circ}$ ano $(\mathrm{m})=-2,02+1,81 \mathrm{P}_{\text {foliar}}$, $\mathrm{R}^{2}=40.7 \%$ ]; [Incremento Ht $1^{\circ}$ ano $(\mathrm{m})=-0,55+1,31 \mathrm{P}_{\text {foliar }}, \mathrm{R}^{2}=37 \%$ ]. Os resultados indicam que é imprescindível a adequação dos teores de $\mathrm{P}$ antes da implantação da cultura, confirmando a maior exigência do álamo em solo comparativamente ao Pinus (Garicoitz, 1990; Reissman e Wisniewski, 2000; Gonçalvez et al., 2001).

Assim como N e P, os níveis de $\mathrm{K}$ foliar não foram afetados pela adubação nitrogenada, estando em níveis adequados para o desenvolvimento das plantas, conforme valores indicados por Stanturf et al. (2001). Os teores foliares de K no sítio CA foram superiores ao sítio SO nos dois anos avaliados, correspondendo com os níveis do solo, em que, no SO, os teores também foram menores que no CA, indo de $0,1 \mathrm{a} 0,2 \mathrm{cmol}_{\mathrm{c}}$ $\mathrm{dm}^{-3}$ contra níveis de 0,25 a $0,61 \mathrm{cmol}_{\mathrm{C}} \mathrm{dm}^{-3}$. Não foi observada, contudo, nenhuma relação entre os teores de $\mathrm{K}$ e o crescimento em CAP e altura total.

O tratamento com NPK também não afetou os teores foliares de $\mathrm{K}$, dado possivelmente pelo solo apresentar teores adequados. Por outro lado, a dose pode ter sido insuficiente para provocar aumento do K foliar. Esses resultados sugerem que o K não está sendo um fator limitante ao desenvolvimento do álamo, mesmo sem adição deste (Jobling, 1990; Brown, 1999).

Ao contrário dos teores de $\mathrm{P}$, os teores foliares de $\mathrm{S}$ encontrados no segundo ano estiveram muito altos, variando de 4,3 a 4,9 $\mathrm{g} \mathrm{kg}^{-1}$ em CA e 4,7 a 5,0 $\mathrm{g} \mathrm{kg}^{-1}$ em SO, quando comparado à faixa de concentração de 1 a $2 \mathrm{~g} \mathrm{~S} \mathrm{~kg}^{-1}$ citado como adequados para as plantas (Malavolta, 1989; Marschner, 1995). Confirmando os elevados valores de S na folha Brown e Van den Driessche (2002) observaram valores médios de $1,1 \mathrm{~g} \mathrm{~kg}^{-1}$ para o segundo ano e até $1,5 \mathrm{mg} \mathrm{kg}^{-1}$ para o primeiro ano na cultura do álamo. Todavia, não foram observados sintomas visuais de deficiência nutricional ou toxidez causado por desbalanço dado ao elevado teor de $\mathrm{S}$ na plantas sugerindo que tais valores estejam relacionados à condição de solo e planta na região.

Os teores de Ca foliar variaram, no CA, de 9,76 a 12,27 e 9,9 a 12,6 $\mathrm{g} \mathrm{kg}^{-1}$, para primeiro e segundo anos respectivamente e, no SO, foram de 5,18 a 6,44 e 6,3 a 7,0 $\mathrm{g} \mathrm{kg}^{-1}$, para primeiro e segundo anos respectivamente. Esses teores se mostraram diretamente relacionados com o incremento em CAP [Incremento CAP $1^{\circ}$ ano $(\mathrm{cm})=-0,07+0,42 \mathrm{Ca}_{\text {foliar, }} \mathrm{R}^{2}=65 \%$; [Incremento CAP após 2 anos $(\mathrm{cm})=3,44$ $+0,58 \mathrm{Ca}_{\text {foliar, }}, \mathrm{R}^{2}=46 \%$ ] e altura da planta, nos dois anos avaliados, de modo isolado ou em combinação com P [Incremento CAP $1^{\circ}$ ano $(\mathrm{cm})=-5,45+0,32 \mathrm{Ca}_{\text {foliar }}+4,78 \mathrm{P}_{\text {foliar, }} \mathrm{R}^{2}=69 \%$ ]; [Incremento CAP após 2 anos CAP seg. ano $(\mathrm{cm})=-1,08+0,52 \mathrm{Ca}_{\text {foliar }}+3,69 \mathrm{P}_{\text {foliar, }}, \mathrm{R}^{2}=60 \%$ ]; [Incremento Ht após 2 anos $(\mathrm{m})=-1,17+0,11 \mathrm{Ca}$ foliar $+1,05 \mathrm{P}_{\text {foliar }}, \mathrm{R}^{2}=67 \%$ ], sendo esses os dois fatores mais limitantes para $\mathrm{o}$ desenvolvimento da cultura.

O efeito do Ca sobre crescimento pode estar relacionado à alta acidez do solo, sobretudo no SO, que apresenta V\% de 13 e pH de 4,0, embora os teores de Ca no solo sejam razoáveis (2,6 $\left.\mathrm{cmol}_{\mathrm{C}} \mathrm{dm}^{-3}\right)$, sugerindo a relação desse elemento à acidez do solo. Variações de $\mathrm{pH}$ observadas mostraram uma relação linear com o desenvolvimento das plantas (Incremento em CAP após 2 anos $(\mathrm{cm})=-14,7+5,44 \mathrm{pH}, \mathrm{R}^{2}=85 \%$ ), para $\mathrm{pH}$ entre 3,8 e 5,1, tanto para a CAP como para Ht (Incremento em Ht após 2 anos $(\mathrm{m})=-3,85+1,19 \mathrm{pH}, \mathrm{R}^{2}=$ 76\%) , concordando com Jobling (1990), Brown (1999) e Dickmann (2001), indicando que o álamo é uma cultura sensível ao $\mathrm{pH}$, necessitando de níveis elevados para o seu bom desenvolvimento. Contudo, Brown e 
van den Driesshe (2002), trabalhando com álamos em solos com pH 4,9 e 6,0, valores esses superiores ao existente nos solos do presente trabalho, obtiveram correlação negativa para o crescimento em volume. Mesma resposta foi observada por Medeiros e Hope (2002) em vasos sob condição controlada. Tal diversidade de resultado pode estar relacionados com faixa de $\mathrm{pH}$ trabalhado, tipo de solo, clima e clone utilizado.

O elevado teor de matéria orgânica tem possibilitado uma grande tolerância das plantas a baixo pH, contanto que haja uma disponibilidade de Ca adequada e também porque o $\mathrm{Al}$, embora em saturação alta, não apresenta teores tão elevados em solução, comparativamente aos solos minerais (Evans e Kamprath, 1970). Tal fato tem propiciado produções elevadas mesmo com pH 4,2 em solos turfosos (Millar, 1959). Por outro, lado o elevado poder tampão da matéria orgânica dificulta a elevação do $\mathrm{pH}$, e são necessárias grandes quantidades de corretivo (Canellas et al., 1999). Portanto, para os solos estudados, fica evidente a necessidade de uma adequação do $\mathrm{pH}$ mesmo para solos orgânicos para a implantação de povoamentos de álamo, considerando a sua elevada acidez.

Os teores foliares de Mg variaram de 3,1 a 3,9 e 3,2 a 3,6 $\mathrm{g} \mathrm{kg}^{-1}$ para primeiro e segundo anos respectivamente em CA e de 3,9 a 4,4 e 3,7 a 4,1 $\mathrm{g} \mathrm{kg}^{-1}$ para primeiro e segundo anos respectivamente em SO, não sendo afetados pelo tratamento. Tais valores estão muito aquém dos $15 \mathrm{~g} \mathrm{~kg}^{-1}$ indicado por Fiedler et al. (1973) como nível adequado para álamo.

\section{Teores foliares micronutrientes (Boro, Manganês, Ferro, Cobre e Zinco).}

Os resultados obtidos mostram que não houve alteração do teor foliar de B em função da adubação. Contudo, constatou-se uma grande diferença entre os teores foliares do álamo cultivado em SO e CA (Tabela 3). A concentração foliar desse elemento foi maior no CA, variando de 32,2 a 37,5 mg kg-1 nos dois anos avaliados, valores esses considerados normais para a maioria das plantas (Marshner, 1995). Já, no SO esses teores variaram de 9,5 a 14,5 mg. kg-1 ${ }^{-1}$ indicando uma provável deficiência, apesar de não ter sido detectados sintomas visuais.

TABELA 3: Teores foliares de $\mathrm{B}\left(\mathrm{mg} \mathrm{kg}^{-1}\right)$ em álamo para primeiro e segundo anos em relação às diferentes doses de adubação.

TABLE 3: Poplar B foliar concentration for first and second years in relation to different fertilization levels.

\begin{tabular}{l|c|c|c|c|c|c|c}
\hline Época & Sítio & $80 \mathrm{~N}$ & $40 \mathrm{~N}$ & $25: 17: 11 \mathrm{NPK}$ & $20 \mathrm{~N}$ & $00 \mathrm{~N}$ & Média \\
\hline $1^{\circ}$ ano & CA & 32,2 & 35,4 & 34,0 & 35,0 & 32,2 & 33,8 \\
& SO & 9,5 & 14,1 & 11,2 & 11,8 & 13,1 & 11,9 \\
\hline $2^{\circ}$ ano & CA & 36,6 & 36,7 & 37,0 & 32,7 & 37,5 & 36,1 \\
& SO & 14,3 & 13,4 & 14,3 & 14,2 & 12,4 & 13,7 \\
\hline
\end{tabular}

Em que: $\mathrm{CA}$ = cambissolo; $\mathrm{SO}$ = solo orgânico.

Apesar de haver uma relação direta entre teor de matéria orgânica do solo e disponibilidade de B, deficiência de B em solos orgânicos e turfa tem sido constatada (Prasad e Byrne, 1975; Quaggio e Ramos, 1987).

A ocorrência de sintomas de deficiência de B, como encarquilhamento de folhas novas e perda de crescimento foram detectados e corrigidos com aplicação no viveiro implantado em solo de várzea do Iguaçu (Dados das Ind. Andrade Latorre S/A). Ainda, perda de dominância apical tem sido observada em algumas condições sugerindo a ocorrência de deficiência de B nos solos de várzea do rio Iguaçu.

Ao contrario do que foi observado na folha, no solo, os valores desse nutriente variaram de 0,23 a $0,35 \mathrm{mg} \mathrm{dm}^{-3}$ no SO e de 0,28 a $0,58 \mathrm{mg} \mathrm{dm}^{-3}$ para CA (Tabela 1 ), sendo esses valores considerados normais (Raij et al., 1996). No CA, os teores foliares mais altos se deram, além dos maiores teores do solo, provavelmente pela maior condição de enraizamento que esse solo proporciona, conforme já comentado. Inversamente, no SO, a absorção foi dificultada pelo baixo $\mathrm{pH}$, que provavelmente dificultou o crescimento radicular, associado a solos mais rasos (observado a campo).

O B foliar, junto com $\mathrm{Ca}$, indicou efeito sobre a altura das plantas no segundo ano avaliado, confirmando seu efeito na formação de gemas de crescimento no segundo ano [Incremento Ht após 2 anos (m) $\mathrm{SO}=-0,936+0,16 \mathrm{Ca} \mathrm{a}_{\text {foliar }}+0,069 * \mathrm{~B}_{\text {foliar }}, \mathrm{R}^{2}=68 \%$ ). Esses resultados indicam a necessidade de aplicação periódica de B, sobretudo considerando-se que há a necessidade de elevação do pH. 
A adubação não alterou os teores de Mn elemento na planta. Mas assim como B, grande diferença na concentração foliar foi observada entre os solos. Os maiores teores foliares de Mn ocorreram no SO, com 275 e $355 \mathrm{mg} \mathrm{kg}^{-1}$ para primeiro e segundo ano respectivamente, contra 169 e $197 \mathrm{mg} \mathrm{kg}^{-1}$ do CA para primeiro e segundo ano respectivamente. Os teores foliares de Mn em CA são normais, enquanto que os do SO estão altos.

Inversamente aos seus teores foliares, os teores de Mn extraído do solo SO foram muito inferiores aos do CA (2,8 a 6,0 $\mathrm{g} \mathrm{dm}^{-3}$ contra 19,6 a 122,4 $\mathrm{g} \mathrm{dm}^{-3}$ ). Dificuldade na determinação da disponibilidade de Mn do solo via extração química vem sendo reportada em diferentes condições (Shuman, 1980; Pavan e Myiazawa, 1983). Ainda, a disponibilidade desse elemento é provavelmente muito variável no perfil do solo, dado a condição de má drenagem no perfil, especialmente no SO, visto que condição de redução pode elevar a disponibilidade de Mn (Ponnamperuma, 1987). Logo, a coleta de solo a $20 \mathrm{~cm}$ de profundidade não representa a disponibilidade do elemento para a planta. Melhores resultados foram obtidos quando se relacionou a produtividade da soja com o resultado da extração química de Mn das camadas inferiores a 15 cm (Robertson et al., 1973).

Constatou-se uma correlação inversa entre os teores de Mn foliar e os incrementos em CAP para o primeiro ano avaliado [Incremento $1^{\circ}$ ano CAP $(\mathrm{cm})=6,17-0,013 \mathrm{Mn}_{\text {foliarlano, }} \mathrm{R}^{2}=40 \%$ ] e $\mathrm{Ht}$, indicando que possivelmente esteja havendo um efeito fitotóxico do elemento, resultante do baixo $\mathrm{pH}$ e ambiente de redução. Sugerindo assim que os valores de Mn encontrado na folha estavam em níveis muito altos para a cultura. Também esse efeito pode ser indireto, em função do pH, pois há uma correlação inversa entre $\mathrm{pH}$ do solo e teor foliar de $\mathrm{Mn}$ (Teor foliar $\mathrm{Mn} 2^{\circ}$ ano $=9233,15-3861,9 \mathrm{pH}+409,7 \mathrm{pH}^{2}, \mathrm{R}^{2}=78 \%$ ). Logo, adequação da acidez via calagem poderá ser indicada para diminuição dos teores foliares, assim como fora observado em outras culturas por Melsted et al. (1963), Jackson et al. (1966), Assmann, (1995), Quaggio et al. (1998), Caires et al. (2003). Todavia, calagem isolada pode não ser a solução, caso o problema seja com excesso de água no solo, havendo necessidade de uma drenagem mais eficiente.

A adição de $\mathrm{N}$ também não afetou a concentração de $\mathrm{Fe}$, $\mathrm{Zn}$ e $\mathrm{Cu}$. Esses elementos não tiveram correlação com o crescimento. Os teores foliares de Fe variaram de 39 a 49 mg kg-1 e 78 a 90 mg kg-1 para primeiro e segundo anos respectivamente em CA e 37 a $56 \mathrm{mg} \mathrm{kg-}^{-1}$ e 60 a $67 \mathrm{mg} \mathrm{kg-1} \mathrm{em} \mathrm{SO} \mathrm{para} \mathrm{primeiro} \mathrm{e}$ segundo anos respectivamente.

Para o $\mathrm{Cu}$ os teores foliares variaram de 6,3 a 9 mg kg-1 e 9,3 a 10,3mg kg-1 em CA para primeiro e segundo ano respectivamente e para SO variou de 6,3 a 6,85 e 10,7 a 12,7 mg.kg-1 ${ }^{1}$ para primeiro e segundo ano respectivamente.

\section{CONCLUSÕES}

Os plantios de álamo em solos de encosta apresentaram um desenvolvimento superior aos de várzea, contrariando a idéia de que aquele solo seria o ideal para a cultura.

A adição de $\mathrm{N}$ teve pequeno efeito sobre o crescimento das plantas de álamo nas condições do trabalho, dados provavelmente à elevada capacidade de suprimento do solo e/ou ocorrência de fatores limitantes.

Os baixo níveis de $\mathrm{P}$ e relação N/P mostraram que $\mathrm{P}$ foi um dos fatores limitantes, alem do B. Assim, confirma-se a exigência do álamo quanto à fertilidade do solo, sendo necessário a adequação do $\mathrm{pH}$ (calagem) e P antes do estabelecimento da cultura e suprimento continuo de B.

\section{REFERÊNCIAS BIBLIOGRÁFICAS}

ASSMANN, T. S. Influência da aplicação do calcário Irati (São Mateus do Sul - PR) mo teores de S, Cu, Fe, e Zn no solo e na planta de milho. Curitiba, 1995. Dissertação (Mestrado em Ciência do Solo) - Universidade Federal do Paraná, Curitiba, 1995.

BAUM C; MAKESCHIN F. Effects of nitrogen and phosphorus fertilization on mycorrhizal formation of two poplar clones (Populus trichocarpa and P. tremula x tremuloides). Journal Of Plant Nutrition And Soil Science-Zeitschrift Fur Pflanzenernahrung Und Bodenkunde, v. 163, n.5, p.491-497, 2000.

BELANGER, C.; MANION, P. D.; GRIFFIN, D.H. Aminoacid content of water stressed plantlets of Populus tremuloides clones in relation to clonal susceptibility to Hypoxylon mammatum in vitro. Ca. J. Bot., v. 68, p.66-29, 
1990.

BIRLER. A. S. A study of yields from “I-214” poplar plantations. Izmit 1994. 115p. (Miscellaneous publication series n. 5).

BROWN, K. R.; DRIESSCHE, R. van den. Growth and nutrition of hybrid poplars over 3 years at fertilization at planting. Canadian Journal of Forest Research, Victoria, v. 32, p 226-232, 2002.

BROWN, K. R. Mineral nutrition and fertilization of deciduous broadleaved trees species in British Columbia. Victoria : Research Branch. B. C. Ministry of Forests, 1999. 42p. (Working Paper; 42)

CAIRES, E. F.; BLUM, J.; BARTH, G.; GARBUIO, F. J.; KUSMAN, M. T. Alterações químicas do solo e resposta da soja ao calcário e gesso aplicados na implantação do sistema plantio direto. R. Bras. Ci. Solo. Campinas, v. 27, p. 275286, 2003.

CANELLAS, L. P.; SANTOS, G.A.; AMARAL SOBRINHO. Reações da matéria orgânica. In: SANTOS, G. A. ; CAMARGO, F. A. O. Fundamentos da matéria orgânica do solo: ecossistemas tropicais e subtropicais. Porto Alegre, Gênesis, 1999. 508p. Parte I, Cap. 5.

DICKMANN, D. I.; ISEBRANDS, J. G.; BLAKE, T. J.; KOSOLA, K; KORT, J. Physiological ecology of poplars. In: DICKMANN, D. I. ; ISEBRANDS, J. G. ; ECKENWALDER, J. E ; RICHARDSON, J. Poplar Culture In North America. Ottawa : NRC Research Press, 2001. Part A, Chapter 1, p. 1-42. (National Research Council of Canada, ON K 1 A 0R6)

DRIESSHE, R. van den. Phosphorus, copper and zinc supply levels influence growth and nutrition of a young Populus trichocarpa (Torr. and Gray) x P. deltoides (Bartr. ex. Marsh.) hybrid. New For., v. 19, p. 143-147, 2000.

ENTE NAZIONALE PER LA CELLULOSA E PER LA CARTA. Pioppicoltura. Roma, 1987. 70p.

ERICSSON, T.; RYTTER, L.; LINDER, S. Nutritional dynamics and requirements of short rotation forests. In: MITCHELL, C.P., FORD-ROBERTSON, J.B., HINCKLEY, T. ; SENNERBY-FORSSE, L. (eds.) Ecophysiology of Short Rotation Forest Crops. London : Elsevier Applied Science, 1992. p. 35-65.

EVANS, C. E.; KAMPRATH, E. J. Lime response as related o percent Al saturation, solution Al, and organic matter content. Soil Sci. Soc. Amer. Proc., v. 34, p. 893-896, 1970.

FIEDLER, H. J.; NEBE, W.; HOFFMANN, F. Forstliche pflanzenernährung und düngung. Stuttgart : Gustav Fischer, 1973. 481p.

GARICOITS, L.S.L. Estado nutricional e fatores do solo limitantes do crescimento de P. taeda L. em Telêmaco Borba (PR). Curitiba,1990. Dissertação (mestrado) - Universidade Federal do Paraná, Curitiba, 1990.

GONÇALVEZ, J. L. M.; BARROS, N. F.; NAMBIAR, E. K. S.; NOVAIS, R. F. Soil and stand management for short rotation plantations. In: NAMBIAR, E. K. S. ; BROWN, A. G. Management of soil, nutrients and water in tropical plantations forests. Canberra: ACIAR, 1997. 571p.

GONÇALVES, J. L. M. Recomendações de adubação para Eucaliptus, Pinus e espécies nativas. Piracicaba: IPEF, 2001. (Documentos Florestais)

HARVEY H. P.; DRIESSCHE, van den. Nutrition, xilem cavitation and drought resistance in hybrid poplars. Tree Physiology, v. 17, p. 647-654, 1997.

JACKSON, T. L.; WESTRMAN, D. T.; MOORE, D.P. The effect of chloride and lime on the manganese uptake by bush beans and sweet corn. Soil Sci. Soc. Am. J., Madison, v. 30, p. 70-73.

JOBLING, J. Poplars for wood production and amenity. London : Forestry Commission : HSMO Publications, 1990. p.38-52. (Forestry Commission Bulletin ; 92)

JOHNSON, J. D.; ZHAO, S. Nitrogen use efficiency and productivity of hybrid poplars: clonal differences related to parentages. INTERNATIONAL POPLAR COMMISSION, 22., 2004. Abstracts of submitted papers. 2004.

MALAVOLTA, E. Avaliação do estado nutricional de plantas: princípios e aplicações. Piracicaba : Associação Brasileira para a Pesquisa da Potassa e do Fosfato, 1989. 201p.

MARSCHNER, H. Mineral nutrition of higher plants. San Diego : Academic Press, 1995. 889p.

MEDEIROS, J. G. S.; HOPPE, J. M. Efeito da aplicação de calcário em estacas de Populus deltoides Bart. ex Marsh cultivadas em vaso. Ciência Florestal, Santa Maria, v. 12, n 2, p 161-167, 2002

MILLAR, C. E. Soil fertility. New York : John Wiley, 1959.

OOSTEN, C. Van; Zabek, L. M. Fertilization of short rotation instensive cultures (SRIC) hybrid poplars plantation in 
southwestern British Columbia, Canada. INTERNATIONAL POPLAR COMMISSION, 22., 2004. Abstracts of submitted papers. 2004.

PAVAN, M. A.; MIYAZAWA, M. Disponibilidade de manganês no solo: dificuldades e problemas na interpretação da análise para fins de fertilidade. R. Bras. Ciência Solo, Campinas, v. 3, p. 155-178, 1983.

PRASAD, M.; BYRNE, E . Boron source and lime effects on the yield os three crops grown in peat. Agron. Journal, v. 67, p. 553-556, 1975.

PONNAMPERUMA, F.N. Electrochemical changes in sumerged soils and the growth of rice. In: BRADY, N.C. Soils and rice. Los Banos : International Rice Research Institute, 1987. p.421-441.

QUAGGIO, J.A; RAMOS, V.J. Resposta da batata à calagem e Boro. R. Bras. Ciência Solo, Campinas, v. 10, p. 247- 251, 1987.

QUAGGIO, J. A.; GALLO; P. B. FURLANI; A. M. C.; MASCARENHAS H. A. Isoquantas de produtividade de soja e sorgo para níveis de calagem e molibdênio. R. Bras. Ci. Solo, v. 22, p. 334-337, 1998.

RAIJ, B. Van; CANTARELLA, H.; QUAGGIO, J. A.; e FURLANI, A. M. C. (eds.) Recomendações de adubação e calagem para o Estado de São Paulo. 2.ed. Campinas : Instituto Agronômico e Fundação IAC, 1996. 285p. (Boletim Técnico ; 100)

RAIJ, B. van; QUAGGIO, J.A. Métodos de análise de solo para fins de fertilidade. Campinas: Instituto Agronômico, 1983. 31p. (IAC Boletim Técnico ; 81)

REISSMANN, C.B.; WISNIEWSKI, C. Aspectos nutricionais de plantios de Pinus. In : GONCALVES, J. L. de M.; BENEDETTI, V. Nutrição e fertilização florestal. Piracicaba: IPEF, 2000. p.136-162.

ROBERTSON, W. K.; THOMPSOM, L.G.; MARTIN, F. G. Manganese and Copper requirements for soybeans. Agronomy Journal, Madison, v. 65 , p. 641-644, 1973.

ROCHA, H. O; RAUEN, M. J.; CARDOSO, A. Levantamento pedológico das Fazendas São Joaquim e São João Batista, São Mateus do Sul, Paraná. 1999. 200p. Acervo das Indústrias Andrade Latorre S/A.

SHUMAN, L.M. Effect of soil temperature, moisture, and air-drying on extractrable manganese, iron, copper, and zinc. Soil Science, Baltimore, v. 30, n. 6, p. 336-343, 1980.

STANTURF, J. A.; Van OOSTEN, C.; NETZER, D. A.; COLEMAN, M. D.; PORTWOOD, C. J. Ecology and silviculture of poplar plantations. In: DICKMANN, D. I.; ISEBRANDS, J. G.; ECKENWALDER, J. E.; RICHARDSON, J. (eds.) Poplar Culture In North America. Ottawa : National Research Council of Canada, 2001. Part A, Chapter 1, p.1-42. 